05,08

\title{
Намагничивание парамагнитных ионов фазово-модулированными СВЧ-импульсами в нулевом магнитном поле
}

\author{
(С) М.Р. Арифууллин, В.Л. Бердинский \\ Оренбургский государственный университет, \\ Оренбург, Россия \\ E-mail: arifullinm@mail.ru
}

Поступила в Редакцию 3 октября 2019 г.

В окончательной редакции 3 октября 2019 г.

Принята к публикации 30 октября 2019 г.

\begin{abstract}
Теоретически предсказан эффект создания намагниченности парамагнитных ионов со спином $S=1$ в твердом теле в нулевом магнитном поле путем импульсных манипуляции электронными спинами. Для этой цели предлагается использовать скрещенные СВЧ-импульсы с различной начальной фазой или импульсы конечной длительности. Описаны резонансные случаи, когда частота СВЧ-импульсов совпадает с частотой расщепления уровней в нулевом поле. Показано сходство данного эффекта с переходом „выстраиваниеориентация“, который наблюдался в оптических экспериментах.
\end{abstract}

Ключевые слова: СВЧ-импульсы, парамагнитные ионы, кубит, расщепление в нулевом поле.

DOI: 10.21883/FTT.2020.03.49002.604

\section{1. Введение}

Очевидно, что в нулевом магнитном поле высокоспиновые парамагнитные ионы с суммарным спином $S \geq 1$, такие как $\mathrm{Ni}^{2+}, \mathrm{Fe}^{3+}, \mathrm{Cr}^{2+}, \mathrm{Gd}^{3+}$ и другие, создают нулевую намагниченность. В твердом теле их спиновые состояния определяется кристаллическим полем или полем лигандов и спин-орбитальным взаимодействием. В нулевом магнитном поле спиновые состояния ионов с двумя неспаренными электронами и с суммарным спином $S=1$ в кристаллах с одноосной анизотропией описываются спиновым гамильтонианом [1]:

$$
H_{0}=D \cdot S_{Z}^{2},
$$

где $D$ - параметр расщепления в нулевом поле, который определяется спин-орбитальным взаимодействием. Собственными состояниями этого гамильтониана являются спиновые векторы $\left|T_{0}\right\rangle=2^{-1 / 2}\left|\alpha_{i} \beta_{j}+\beta_{i} \alpha_{j}\right\rangle=|0\rangle$, $|A\rangle=2^{-1 / 2}\left|\alpha_{i} \alpha_{j}-\beta_{i} \beta_{j}\right\rangle=2^{-1 / 2}(|1\rangle-|-1\rangle)$ и $|B\rangle=$ $=2^{-1 / 2}\left|\alpha_{i} \alpha_{j}+\beta_{i} \beta_{j}\right\rangle=2^{-1 / 2}(|1\rangle+|-1\rangle)$. При низких температурах $k T \ll D$ населенным оказывается нижнее спиновое состояние $|0\rangle$. Векторы $|A\rangle$ и $|B\rangle$ являются суперпозициями состояний $\left|T_{+}\right\rangle=\left|\alpha_{1} \alpha_{2}\right\rangle=|+1\rangle$ и $|T\rangle=\left|\beta_{1} \beta_{2}\right\rangle=|-1\rangle$ с проекциями спина $S_{Z}= \pm 1$. Очевидно, что это белловские запутанные состояния, образующиеся естественным образом в высокоспиновых парамагнитных ионах. Здесь следует отметить, что в нулевом магнитном поле все три спиновых состояния $|0\rangle,|A\rangle$ и $|B\rangle$ не создают магнитных моментов, так как средние значения всех спиновых операторов $\left(\sigma_{1 i}+\sigma_{2 i}\right)$ $(i=x, y, z)$ равны нулю. Следовательно, для таких ионов в кристаллической решетке отсутствует магнитнодипольное взаимодействие, которое приводит к дополнительному механизму декогеренции. Эти же состояния реализуются в триплетных органических молекулах, однако, для этих органических молекул расщепление в нулевом поле обусловлено не спин-орбитальным взаимодействием, а диполь-дипольным взаимодействием [2]. В органических молекулах при низких температурах времена жизни электронных спинов в этих состояниях могут быть достаточно большими, порядка нескольких десятков секунд [3-4]. Времена спин-решеточной релаксации парамагнитных ионов в нулевом магнитном поле необычайно продолжительны и составляют порядка миллисекунд и более [5], что согласуется с теориями, изложенными в работах [6]. Достаточно длительные времена спиновой релаксации наблюдались в металлоорганических соединениях и комплексах парамагнитных ионов, синтезированных для создания молекулярных магнетиков и мономолекулярных магнитов [7-10]. Эти результаты делают такие парамагнитные ионы и триплетные органические молекулы чрезвычайно привлекательными для использования в качестве элементной базы квантовых компьютеров [11] даже несмотря на то, что увеличение времени спиновой релаксации влияет на скорость считывания информации.

Даже в отсутствие внешнего магнитного поля (в нулевом магнитном поле) диполь-дипольное и спинорбитальное взаимодействия устраняют вырождение уровней с суммарным спином $S_{Z}=0$ и $S_{Z}= \pm 1[1]$. В зависимости от типа ионов и кристаллической решетки расщепление в нулевом поле, как правило, попадает в технически доступные радиочастотные или микроволновые диапазоны электромагнитного поля. Магнитная компонента таких полей способна индуцировать переходы между энергетическими уровнями с различными значениями $S_{Z}$. Ранее кристаллы с примесными высокоспиновыми ионами в нулевом магнитном поле были предложены в качестве активных сред для микроволно- 
вых мазеров [12-13]. Создание таких мазеров сопровождалось развитием радиотехнических методов управления спиновыми состояниями ионов. Активными примесными ионами могли быть ионы $\mathrm{Ni}^{2+}, \mathrm{Fe}^{3+}, \mathrm{Cr}^{2+}, \mathrm{Gd}^{3+}$ и другие лантаноиды с различными наборами спиновых и энергетических уровней. Очевидно, что применение низких температур позволяет управлять исходными населенностями спиновых подуровней и временами спиновой релаксации и декогеренции.

В термодинамическом равновесии возбужденные уровни $|A\rangle$ и $|B\rangle$ равнонаселены; это состояние аналогично явлению оптического выстраивания орбитального углового момента атомов [14] и экситонов [15]. Оптическое выстраивание - это одинаковое заселение состояний с проекциями орбитального момента $L_{Z}= \pm 1$, при этом макроскопический магнитный момент равен нулю. Для создания макроскопического магнитного момента необходимо преимущественное заселение состояний с определенным значением проекции $L_{Z}$. Во многих работах, например, [16], описано явление перехода оптического выстраивания в поляризацию, что детектировалось по изменению степени поляризации спонтанного излучения. Для парамагнитных ионов с электронным спином $S=1$ аналогом оптического выстраивания является состояние с равными населенностями состояний $|A\rangle$ и $|B\rangle$. Радиочастотные или СВЧ-импульсы позволяют манипулировать населенностями этих состояний и когерентными соотношениями между ними и, следовательно, магнитными свойствами ионов. Для спиновых подуровней парамагнитных ионов переход выстраивания в поляризацию, управляемый радиочастотными импульсами, должен сопровождаться появлением макроскопической намагниченности даже в нулевом магнитном поле.

Цель данной работы - изучение спиновой динамики парамагнитных ионов со спином $S=1$ в нулевом магнитном поле и возможностей создания электронной намагниченности в результате формирования когерентной суперпозиции спиновых состояний $\left|S_{Z}=1\right\rangle$ и $\left|S_{Z}=-1\right\rangle$ с помощью радиочастотных или СВЧ-импульсов с различными фазами и поляризациями.

\section{2. Спиновая динамика ионов со спином $S=1$}

Для описания и анализа действия СВЧ-импульсов на парамагнитные ионы спиновый гамильтониан (1) удобно записать через операторы Паули отдельных электронов. Поскольку,

$$
\begin{aligned}
S_{Z}^{2} & =\left(\sigma_{1 Z}+\sigma_{2 Z}\right)^{2} / 4=\left(2+2 \sigma_{1 Z} \sigma_{2 Z}\right) / 4 \\
& =1 / 2+\sigma_{1 Z} \sigma_{2 Z} / 2,
\end{aligned}
$$

To

$$
H_{0}=\frac{1}{2} D \cdot \sigma_{1 Z} \sigma_{2 Z}
$$

собственными состояниями которого являются те же самые векторы $|0\rangle,|A\rangle$ и $|B\rangle$, а также вектор синглетного спинового состояния $\left|S_{0}\right\rangle=2^{-1 / 2}\left|\alpha_{i} \beta_{j}-\beta_{i} \alpha_{j}\right\rangle$. Однако это синглетное состояние можно не учитывать в дальнейших расчетах, так как любые операторы поворота реализуют элементы группы $\mathrm{SU}(2)$, а синглетные и триплетные состояния принадлежат разным неприводимым представлениям этой группы. Это означает, что никакие СВЧ-импульсы не способны переводить триплетные состояния в синглетные и обратно.

Для операторов $U(t)$, описывающих действие СВЧполя с частотой $\omega_{G}$ на высокоспиновые ионы, можно получить формулы, удобные для последующих применений в виде полиномов односпиновых операторов Паули. Гамильтониан спиновой системы ионов, взаимодействующих с СВЧ-полем, поляризованным вдоль оси ОХ,

$$
\begin{aligned}
H & =H_{0}+H_{X}(t) \\
& =\frac{1}{2} D \cdot \sigma_{1 Z} \sigma_{2 Z}+g \beta \cdot B_{X}\left(\sigma_{1 X}+\sigma_{2 X}\right) \cos \left(\omega_{G} t\right),
\end{aligned}
$$

где $g \beta \cdot B_{X}$ - частота прецессии.

В представлении взаимодействия гамильтониан взаимодействия $\tilde{H}_{X}(t)$ удобно представить в виде суммы двух операторов.

$$
\begin{aligned}
\tilde{H}_{X}(t)= & \exp \left(i H_{0}\right) g \beta \cdot B_{X}\left(\sigma_{1 X}+\sigma_{2 X}\right) \exp \left(-i H_{0} t\right) \cos \left(\omega_{G} t\right) \\
= & g \beta \cdot\left[\exp \left(i H_{0} t\right) B_{X} \sigma_{1 X} \exp \left(-i H_{0} t\right)\right. \\
& \left.+\exp \left(i H_{0} t\right) B_{X} \sigma_{2 X} \exp \left(-i H_{0} t\right)\right] \cos \left(\omega_{G} t\right) .
\end{aligned}
$$

Так как $\left(\sigma_{1 Z} \sigma_{2 Z}\right)^{2}=I_{1} I_{2}$, то

$$
\exp \left( \pm i H_{0} t\right)=\cos \left(\omega_{D} t / 2\right) \pm i \sin \left(\omega_{D} t / 2\right) \sigma_{1 Z} \sigma_{2 Z}
$$

где $\omega_{D}$ - частота переходов между уровнями расщепления в нулевом поле.

При выполнении условий магнитного резонанса $\omega_{G}=\omega_{D}$, преобразовав все члены, получим

$$
\begin{aligned}
\tilde{H}_{X}(t)= & \frac{g \beta \cdot B_{X}}{2}\left(\sigma_{1 X}+\sigma_{2 X}\right)+f\left(2 \omega_{D} t\right)\left(\sigma_{i Y} \sigma_{j Z}\right) \\
& +f\left(2 \omega_{D} t\right) \sigma_{i X}
\end{aligned}
$$

где функция $f\left(2 \omega_{D} t\right)$ будет определять малую поправку к спиновой эволюции типа сдвига Блоха-Зигерта [17], которой в большинстве случаев пренебрегают. Без этих малых слагаемых самая существенная часть гамильтониана $\tilde{H}_{X}$ уже не зависит от времени. Поскольку операторы $\sigma_{1 X}$ и $\sigma_{2 X}$ коммутируют, то оператор эволюции $\tilde{U}\left(\omega_{X} t\right)$ в представлении взаимодействия можно представить в виде произведения односпиновых операторов синхронной эволюции

$$
\begin{aligned}
\tilde{U}\left(\omega_{X} t\right) & =\exp \left[-i \frac{\omega_{X} t}{2}\left(\sigma_{1 X}+\sigma_{2 X}\right)\right] \\
& =\exp \left[-i \frac{\omega_{X} t}{2}\left(\sigma_{1 X}\right)\right] \exp \left[-i \frac{\omega_{X} t}{2}\left(\sigma_{2 X}\right)\right] \\
& =\tilde{U}_{1}\left(\omega_{X} t\right) \tilde{U}_{2}\left(\omega_{X} t\right),
\end{aligned}
$$


где $\omega_{X}=g \beta \cdot B_{X}$ - частота прецессии вокруг оси $O X$. Оператор поворота двухспиновой системы вокруг оси $O X$ на угол $\theta=\omega \tau$ в результате действия СВЧ-импульса длительностью $\tau$ принимает вид

$$
\begin{aligned}
\tilde{U}(\theta)= & U_{1 X}(\theta) \otimes U_{2 X}(\theta)=\cos ^{2} \frac{\theta}{2}-\left(\sigma_{X 1} \sigma_{X 2}\right) \cdot \sin ^{2} \frac{\theta}{2} \\
& -i \sin \frac{\theta}{2} \cos \frac{\theta}{2}\left(\sigma_{X 1}+\sigma_{X 2}\right) .
\end{aligned}
$$

Фактически, эта формула является аналогом представления экспоненциального оператора $\exp \left[-i \theta S_{X}\right]$, описывающего повороты спина $S=1$ на угол $\theta$ вокруг оси $O X$.

Однако общеизвестные формулы для операторов поворота спинов $S \geq 1$ не позволяют легко и просто описать эффекты фазовых сдвигов СВЧ-полей и их влияние на изменение спиновых состояний ионов и высокоспиновых молекул. Для СВЧ-поля, поляризованного вдоль оси $O Y$ с фазовым сдвигом $\delta$ относительно СВЧ-поля, гамильтониан взаимодействия имеет вид

$$
H_{Y}(t)=g \beta \cdot B_{Y}\left(\sigma_{1 Y}+\sigma_{2 Y}\right) \cos \left(\omega_{G} t-\delta\right) .
$$

Если, как и в предыдущем случае, считать, что $\omega_{G}=\omega_{D}$ и пренебречь малыми слагаемыми с удвоенной частотой $2 \omega_{D}$, то в представлении взаимодействия

$$
\tilde{H}_{Y}=\frac{\omega_{Y}}{2}\left[\left(\sigma_{1 Y}+\sigma_{2 Y}\right) \cos \delta+\left(\sigma_{1 X} \sigma_{2 Z}+\sigma_{2 X} \sigma_{1 Z}\right) \sin \delta\right] .
$$

Оператор поворота $U_{Y}(\theta, \delta)$ двухспиновой системы на угол $\theta$ СВЧ-импульсом с фазовым сдвигом $\delta$ приобретает вид

$$
\begin{aligned}
& \tilde{U}_{Y}(\theta, \delta)=\cos ^{2} \frac{\theta}{2}-\sigma_{Y 1} \sigma_{Y 2} \cdot \sin ^{2} \frac{\theta}{2}-i \sin \frac{\theta}{2} \cos \frac{\theta}{2} \\
& \quad \times\left(\left(\sigma_{Y 1}+\sigma_{Y 2}\right) \cos \delta+\left(\sigma_{X 1} \cdot \sigma_{Z 2}+\sigma_{X 2} \cdot \sigma_{Z 1}\right) \sin \delta\right) .
\end{aligned}
$$

Аналогичным образом можно показать, что оператор $U_{X}(\theta, \delta)$ поворота спинов вокруг оси $O X$ имеет вид:

$$
\begin{aligned}
\tilde{U}_{X}(\theta, \delta) & =\cos ^{2} \frac{\theta}{2}-\sigma_{X 1} \sigma_{X 2} \cdot \sin ^{2} \frac{\theta}{2}-i \sin \frac{\theta}{2} \cos \frac{\theta}{2} \\
& \times\left(\left(\sigma_{X 1}+\sigma_{X 2}\right) \cos \delta+\left(\sigma_{Y 1} \cdot \sigma_{Z 2}+\sigma_{Y 2} \cdot \sigma_{Z 1}\right) \cdot \sin \delta\right) .
\end{aligned}
$$

Знание операторов $U_{X}(\theta, \delta)$ и $U_{Y}(\theta, \delta)$ позволяет определить результаты действия СВЧ-импульсов, различающихся амплитудой, поляризацией и фазой, на спиновые состояния парамагнитных ионов со спином $S=1$. Действие оператора $U_{X}(\theta, \delta)$ с произвольным фазовым сдвигом на состояние $\left|T_{0}\right\rangle=2^{-1 / 2}\left|\alpha_{i} \beta_{j}+\beta_{i} \alpha_{j}\right\rangle=|0\rangle$ переводит это состояние в суперпозицию

$$
U_{X}(\theta \delta)|0\rangle=\cos \theta|0\rangle-i \sin \theta \cdot e^{-i \delta}|B\rangle .
$$

Однако, при любых углах поворота $\theta$ и фазах $\delta$ СВЧ-импульсы, поляризованные вдоль оси $O X$, не затрагивают спиновое состояние $|A\rangle$ и

$$
U_{X}(\theta, \delta)|A\rangle=|A\rangle \text {. }
$$

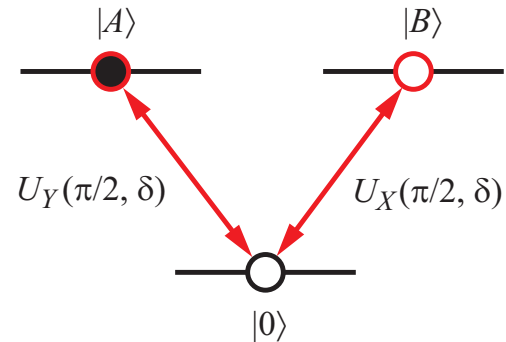

Переходы под воздействием поляризованных СВЧ импульсов. Переход $|0\rangle-|B\rangle$ описывается $\pi / 2$-импульсом, поляризованным вдоль оси $O X$. Переход $|0\rangle-|A\rangle$ описывается $\pi / 2$-импульсом, поляризованным вдоль оси $O Y$.

Если $\theta=\pi / 2$, то СВЧ-импульс $\left.(\pi / 2)\right|_{X}$ переводит спиновую систему из основного запутанного состояния $\left|T_{0}\right\rangle$ в запутанное состояние $|B\rangle$ с фазой $\delta$. В данном случае происходит трансформация фазы $\delta$ СВЧ-импульса в фазу $(-\delta)$ вектора спинового состояния иона.

Если в качестве базисных спиновых функций выбраны состояния $\left|T_{+}\right\rangle=|\alpha \alpha\rangle,\left|T_{-}\right\rangle=|\beta \beta\rangle$, то заселение состояния $|B\rangle$ означает создание выстраивания в системе электронных спинов иона. Очевидно, что при этом не создается ориентация электронных спинов, которая может сопровождаться появлением электронной намагниченности.

Аналогично действие оператора $U_{Y}(\theta, \delta)$ с произвольным фазовым сдвигом на основное состояние $\left|T_{0}\right\rangle$ переводит это состояние в суперпозицию

$$
U_{Y}(\theta, \delta)|0\rangle=\cos \theta|0\rangle-\sin \theta \cdot e^{i \delta}|A\rangle .
$$

Однако СВЧ-импульсы, поляризованные вдоль оси $O Y$, не затрагивают спиновое состояние $|B\rangle$ и

$$
U_{X}(\theta, \delta)|B\rangle=|B\rangle .
$$

Заселение спинового состояния $|A\rangle$ тоже эквивалентно созданию выстраивания в системе электронных спинов иона. Таким образом, импульсные СВЧ-поля с различной поляризацией позволяют селективно заселять спиновые состояния $|A\rangle$ и $|B\rangle$ и таким образом делают их физически различимыми.

Можно показать, что последовательное применения СВЧ-импульсов вдоль осей $O X$ и $O Y$ без фазовых сдвигов позволяет создавать суперпозиции вырожденных состояний

$U_{Y}(\pi / 2,0) U_{X}(\theta, \delta)|0\rangle=(-\cos \theta \cdot|A\rangle-i \sin \theta \cdot|B\rangle)$.

Такая суперпозиция состояний тоже соответствует выстраиванию спиновых моментов электронов с $m=+1$ и $m=-1$, но уже с другими когерентностями.

В одноосном кристалле СВЧ импульсы без сдвига фазы способны формировать выстраивание электронных спинов, но они не создают реального физического магнитного момента иона $M_{Z}$. Далее будет показано, как этот дополнительный фазовый сдвиг может влиять на физическое поведение спина $S=1$. 
Пусть первый импульс $U_{X}(\theta, \delta)$ без фазового сдвига $(\delta=0)$, действующий вдоль оси $O X$ на состояние $|0\rangle$, создает суперпозицию состояний $|0\rangle$ и $|B\rangle$. После этого второй СВЧ-импульс $(\pi / 2)_{Y}$ с фазовым сдвигом $\delta$, действуя на эту суперпозицию, переводит спиновую систему в другую когерентную суперпозицию состояний $|\Psi\rangle$.

$|\Psi\rangle=U_{Y}(\pi / 2, \delta) \cdot U_{X}(\theta, 0)|0\rangle=-\cos \theta e^{i \delta}|A\rangle-i \sin \theta|B\rangle$.

Для такой суперпозиции состояний среднее значение проекции спина $S_{Z}$ и, следовательно, магнитного момента $M_{Z}$ двух электронных спинов иона может быть отлично от нуля

$$
\left\langle S_{Z}\right\rangle=\left\langle\Psi\left|\left(\sigma_{1 Z}+\sigma_{2 Z}\right)\right| \Psi\right\rangle=2 \sin (2 \theta) \sin \delta .
$$

Также легко показать вид матрицы плотности $\rho$ и ее зависимость от длительности импульсов, угла поворота $\theta$ и сдвига фазы $\delta$ в базисе $\left|T_{+}\right\rangle,\left|T_{0}\right\rangle$ и $\left|T_{-}\right\rangle$.

$$
\rho=\frac{1}{2}\left(\begin{array}{ccc}
1+\sin 2 \theta \sin \delta & 0 & -(\cos 2 \theta+i \sin 2 \theta \cos \delta) \\
0 & 0 & 0 \\
-(\cos 2 \theta-i \sin 2 \theta \cos \delta) & 0 & 1-\sin 2 \theta \sin \delta
\end{array}\right) .
$$

Вид этой матрицы плотности наглядно демонстрирует создание разности населенностей состояний $\left|T_{+}\right\rangle$и $\left|T_{-}\right\rangle$ и когерентностей. При любом фазовом сдвиге $\delta \neq 0$ магнитный момент иона $M_{Z}=\neq 0$ достигает максимальных значений при $\theta=\pi / 4$. Отсутствие фазового сдвига в двух последовательных импульсах не создает магнитного момента вдоль оси $Z$. Эффект образования магнитного момента парамагнитных ионов в нулевом магнитном поле СВЧ-импульсами создается сдвигом по фазе одного из импульсов, например, вдоль оси $O Y$. Формирование магнитного момента $M_{Z}=\neq 0$ из когерентной суперпозиции спиновых состояний подобно переходу „выстраивание-ориентация“", которое наблюдалось в оптических экспериментах [16]. Величина намагниченности зависит от концентрации ионов в кристалле и она может оказаться большой величиной.

Измерение магнитного момента иона $M_{Z}$ позволяет идентифицировать состояние спина электронов. Также магнитный момент иона $M_{Z}$ может выступать в качестве физической меры запутанности суперпозиции белловских состояний.

\section{3. Эффекты длительности СВЧ-импульсов}

Можно показать, что импульсы с конечными длительностями $t_{1}$ и $t_{2}$ тоже способны создавать спиновый момент $\left\langle S_{Z}\right\rangle$ также, как и мгновенные импульсы с различной фазой. Для анализа этого эффекта необходимо вернуться в лабораторную систему координат и рассмотреть результат влияния отдельных импульсов конечной длительности без фазовых сдвигов на белловские состояния $|0\rangle,|A\rangle$ и $|B\rangle$

$$
\begin{aligned}
|\Psi\rangle & =\exp \left[-\frac{D t_{1}}{2} \sigma_{1 Z} \sigma_{2 Z}\right] \tilde{U}_{X}(\theta, 0) \exp \left[+\frac{D t_{1}}{2} \sigma_{1 Z} \sigma_{2 Z}\right]|0\rangle \\
& =\cos \theta_{1}|0\rangle-i \sin \theta_{1} \cdot e^{-i \varphi_{1}}|B\rangle
\end{aligned}
$$

где $\varphi_{1}=\omega_{D} \cdot t_{1}=\theta_{1}\left(\omega_{D} / \omega_{p}\right)-$ набег фазы за время $t_{1}$. Импульс вдоль оси $O Y$ создает другую суперпозицию состояний.

$$
\begin{aligned}
\left|\Psi_{2}\right\rangle & =\exp \left[-\frac{D t_{2}}{2} \sigma_{1 Z} \sigma_{2 Z}\right] \tilde{U}_{Y}(\theta, 0) \exp \left[+\frac{D t_{2}}{2} \sigma_{1 Z} \sigma_{2 Z}\right]|0\rangle \\
& =\cos \theta_{2}|0\rangle-i \sin \theta_{2} \cdot e^{-i \varphi_{2}}|A\rangle
\end{aligned}
$$

Импульсы, поляризованные вдоль оси $O Y$, не изменяют фазу состояния $|B\rangle$. После применения последовательных импульсов получим новую суперпозицию состояний

$$
\begin{aligned}
& \left|\Psi_{3}\right\rangle=U_{Y}\left(\theta_{2}, 0\right) U_{X}\left(\theta_{1}, 0\right)|0\rangle=\cos \theta_{1} \cos \theta_{2}|0\rangle \\
& -\cos \theta_{1} \sin \theta_{2} \cdot e^{i \varphi_{2}}|A\rangle-i \sin \theta_{1} \cdot e^{-i \varphi_{1}}|B\rangle=\cos \theta_{1} \cos \theta_{2}|0\rangle \\
& \left.-e^{-i \varphi_{1}}\left(\cos \theta_{1} \sin \theta_{2} \cdot e^{i \delta}|A\rangle\right)+i \sin \theta_{1} \cdot|B\rangle\right)
\end{aligned}
$$

где $\delta=\varphi_{2}+\varphi_{1}-$ фазовый набег. Результат, полученный для мгновенных импульсов, аналогичен формуле (20)

$$
\left\langle S_{Z}\right\rangle=\left\langle\Psi_{3}\left|S_{Z}\right| \Psi_{3}\right\rangle=2 \sin \left(2 \theta_{1}\right) \sin \left(\theta_{2}\right) \sin \delta .
$$

Теперь среднее значение проекции спина иона $\left\langle S_{Z}\right\rangle$ и, следовательно, его магнитный момент полностью определяется длительностью импульсов и фазовым сдвигом. Очевидно, что появление ненулевого спинового момента парамагнитных ионов $\left\langle S_{Z}\right\rangle$ автоматически означает появление ненулевого магнитного момента этих ионов. Величина магнитного момента определяется длительностями импульсов $t_{1}$ и $t_{2}$ и набегами фаз во время действия этих импульсов.

Эффекты образования намагниченности парамагнитных ионов в нулевом магнитном поле в результате действия таких импульсов можно использовать для создания систем квантовой памяти.

\section{4. Заключение}

Теоретически предсказан эффект образования магнитного момента СВЧ-импульсами и фазовым сдвигом одного из импульсов в нулевом магнитном поле для парамагнитных ионов с суммарным электронным спином $S=1$ и с нулевым ядерным спином.

Для парамагнитных ионов с суммарным электронным спином $S=1$, находящихся в нулевом магнитном поле, теоретически предсказан эффект создания магнитного момента $M_{Z} \propto\left\langle S_{Z}\right\rangle \neq 0$ действием СВЧ-импульсов с различными фазовыми сдвигами. 
Предложены СВЧ-импульсы с различной поляризацией и со сдвигом фазы, способные переводить спиновую систему в различные суперпозиционные состояния вырожденных и невырожденных уровней.

Для операторов спиновой эволюции в нулевом магнитном поле и в переменных высокочастотных полях СВЧ-импульсов предложены новые представления экспоненциальных операторов в виде полиномов односпиновых матриц Паули и тригонометрических функций углов поворота спинов.

\section{Финансирование работы}

Работа выполнена при поддержке Российского фонда фундаментальных исследований (грант № 18-37-00374).

\section{Конфликт интересов}

Авторы заявляют, что у них нет конфликта интересов.

\section{Список литературы}

[1] С.А. Альтшулер, Б.М. Козырев. Электронный парамагнитный резонанс соединений элементов промежуточных групп. 2 изд. Наука, М. (1972) 672 с.

[2] А. Керрингтон, Э. Мак-Лечлан. Магнитный резонанс и его применение в химии. Мир, М. (1970). 448 с.

[3] Erinn van Wynsberghe, A. Turak. Candidate Materials as Gain Media in Organic, Triplet-Based, Room-Temperature masers Targeting the ISM Bands / Ed. S. Pyshkin. IntechOpen Limited, London (2017).

[4] J. Breeze, K. Tan, B. Richards, J. Sathian, M. Oxborrow, N.M. Alford. Nature Commun. 6, 6215 (2015).

[5] D.V. Azamat, A.G. Badalyan, A. Dejneka, L. Jastrabik, J. Lančok. Appl. Phys. A 122 (2016).

[6] A. Norambuena, E. Muñ oz, H.T. Dinani, A. Jarmola, P. Maletinsky, D. Budker, J.R. Maze. Phys. Rev. B 97, 094304 (2018).

[7] D.V. Korchagin, A.V. Palii, E.A. Yureva, A.V. Akimov, E.Ya. Misochko, G.V. Shilov, A.D. Talantsev, R.B. Morgunov, A.A. Shakin, S.M. Aldoshin, B.S. Tsukerblat. Dalton Transact. 46, 7540 (2017).

[8] Palii, S. Aldoshin, B. Tsukerblat. J. Phys. Chem. C 121, 27218 (2017).

[9] Palii, S. Aldoshin, B. Tsukerblat, J.J. Borràs-Almenar, J.M. Clemente-Juan, S. Cardona-Serra, E. Coronado. Inorg. Chem. 56, 9547 (2017).

[10] Palii, S. Aldoshin, B. Tsukerblat, J.M. Clemente-Juan, A. Gaita-Ariño, E. Coronado. Phys. Chem. Chem. Phys. 19, 26098 (2017).

[11] M.R. Arifullin, V.L. Berdinskiy. AIP Conf. Proc. (2019). В печати.

[12] A.E. Siegman. Microwave Solid-State Masers. McGraw-Hill. (1964). 583 p.

[13] A.F. Harvey. Microwave engineering. Academic Press, London-N. Y.: (1963) 1313 p.
[14] М.П. Чайка. Оптика и спектроскопия 27 (1969).

[15] Р.И. Джиоев, Б.П. Захарченя, Е.Л. Ивченко, В.Л. Коренев, Ю.Г. Кусраев. ФТТ 40, 5 (1998).

[16] М.П. Чайка. Интерференция вырожденных атомных состояний. Изд-во ЛГУ, Л. (1975). 192 с.

[17] A. Abragam, B. Bleaney. Electron Paramagnetic Resonance of Transition Ions. Clarendon Press, Oxford (1970).

Редактор Т.Н. Василевская 